\title{
中国外来归化植物的编目现状及有关问题
}

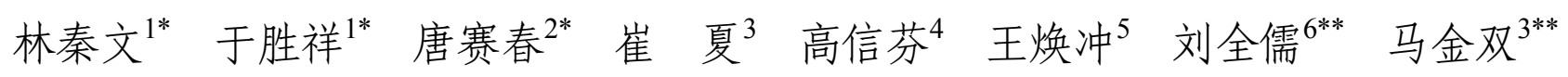
${ }^{1}$ 中国科学院植物研究所, 北京 $100093 ;{ }^{2}$ 广西壮族自治区中国科学院广西植物研究所, 广西桂林 $541006 ;{ }^{3}$ 北京市植物园, 北京 $100093 ;{ }^{4}$ 中国科学院 成都生物研究所, 成都 $610041 ;^{5}$ 云南大学生态与环境学院, 昆明 650091 ; $^{6}$ 北京师范大学生命科学学院, 北京 100875

摘 要 在简要讨论外来植物相关定义的基础上, 对中国外来归化植物的调查和编目现状进行了概述; 并对近年发表的两篇 文章中外来归化植物数据进行了订正。

关键词 外来植物; 归化植物; 入侵植物; 栽培植物; 中国

林秦文, 于胜祥, 唐赛春, 崔夏, 高信芬, 王焕冲, 刘全儒, 马金双 (2021). 中国外来归化植物的编目现状及有关问题. 植物生态学报, 45, 1275-1280. DOI: 10.17521 /cjpe.2021.0314

\section{Current status of naturalized alien species in China and its relative problem}

LIN Qin-Wen ${ }^{1 *}$, YU Sheng-Xiang ${ }^{1 *}$, TANG Sai-Chun ${ }^{2 *}$, CUI Xia ${ }^{3}$, GAO Xin-Fen ${ }^{4}$, WANG Huan-Chong ${ }^{5}$, LIU Quan- $\mathrm{Ru}^{6^{* *}}$, and MA Jin-Shuang $\mathrm{g}^{3 * *}$

${ }^{1}$ Institute of Botany, Chinese Academy of Sciences, Beijing 100093, China; ${ }^{2}$ Guangxi Institute of Botany, Guangxi Zhuang Autonomous Region and Chinese Academy of Sciences, Guilin, Guangxi 541006, China; ${ }^{3}$ Beijing Botanical Garden, Beijing 100093, China; ${ }^{4}$ Chengdu Institute of Biology, Chinese Academy of Sciences, Chengdu 610041, China; ${ }^{5}$ School of Ecology and Environmental Science, Yunnan University, Kunming 650091, China; and ${ }^{6}$ College of Life Sciences, Beijing Normal University, Beijing 100875, China

\section{Abstract}

On the basis of a brief discussion of the definitions of alien plants, we summarized the investigation and research status of China's naturalized alien plants, and made corrections of the data regarding naturalized alien plants on two papers in 2019 and 2021.

Key words alien plants; naturalized plants; invasive plants; cultivated plants; China

Lin QW, Yu SX, Tang SC, Cui X, Gao XF, Wang HC, Liu QR, Ma JS (2021). Current status of naturalized alien species in China and its relative problem. Chinese Journal of Plant Ecology, 45, 1275-1280. DOI: 10.17521/cjpe.2021.0314

自1992年里约热内卢联合国环境与发展大会召 开以来, 生物多样性保护和生态安全保障受到越来 越多的关注, 其中的热点之一即外来物种入侵问 题。近年来, 随着国际贸易不断发展, 对外交流频繁, 国际旅游业迅速升温, 外来物种越来越多地传入中 国, 其中一些外来植物具有入侵性质并成为外来入 侵植物。外来入侵物种本身具有种群优势, 可使本 地物种的生存受到影响甚至灭绝, 破坏物种多样性, 最终导致生态系统破坏, 造成经济损失(夏常英等, 2019)。然而, 外来入侵植物的相关学术研究还有待 提高并加强。

\section{1 引入植物、归化植物、栽培植物、外来入 侵植物的定义}

引入植物(introduced plants)即域外的、非本地 的植物(non-native plants), 经过自然或者人为因素 离开原产地并进入其他领域(一般指国家或地区), 且能够生存并延续的物种; 因其来自异域, 所以又 常被称为外来植物(alien plants)。其中, 能够在自然 条件下自身繁殖(包括有性生殖, 即从种子到种子, 以及孢子繁殖等, 或者通过多年生的习性, 如根或 者茎繁殖等, 即营养繁殖) 并延续的类群, 则称为归 化植物(naturalized plants); 而不能自我繁殖, 需借 助人为因素繁衍的则称为栽培植物(cultivated plants); 归化之后造成危害的(包括经济、生态乃至社会等), 则称为外来入侵植物(invasive alien plants)。这些植物 学领域的基本概念为业界共识, 而且中外无疑(Pyŝek et al., 2004, 2017; Wu et al., 2004; 马金双, 2013, 2014a, 2014b, 2021; 林秦文, 2018; 马金双和李惠茹, 2018; 许光耀等, 2019; 李龙沁, 2021)。

收稿日期Received: 2021-09-01 接受日期Accepted: 2021-11-09

基金项目: 国家自然科学基金(31872645)。Supported by the National Natural Science Foundation of China (31872645).

*同等贡献Contributed equally to this work (Lin QW, linqinwen83@ibcas.ac.cn; Yu SX, yushengxiang@ibcas.ac.cn; Tang SC, tangs@gxib.cn)

**通信作者Corresponding authors (Liu QR, liuquanru@bnu.edu.cn; Ma JS, jinshuangma@gmail.com) 


\section{2 中国外来植物研究领域面临巨大挑战}

一个物种的来源或者说原产地的确定，归根结 底依赖于植物分类学的研究, 特别是最基本的植物 编目工作一一植物志。对于中国而言, 全国性的志书, 如《中国植物志》(中国植物志编委会, 1959-2004) 出版时间间隔比较长, 而且完成时间又比较久远, 很多信息已经更新。另一个全国性工作一一英文版 中国植物志《Flora of China》(Wu et al., 1994-2013) 不仅已经出版10-20多年, 而且因为收载外来植物 相对较少，没有完整地体现中国外来植物的信息。 由于中国植物分类学起步晚、种类多、历史欠账比 较多等原因, 至今没有完全并彻底摸清自己的家底 (马金双, 2014a)。外来植物的研究就更困难, 不仅因 为中国地域辽阔, 自然地理条件复杂, 来自世界很 多地方的植物均可以在中国找到合适的生存环境, 更重要的是, 不知道外来物种是从哪里来的, 何时 进入中国, 又是怎么进来的, 这些外来物种又是一 个什么性质的类群, 它们的生物学特征和生态学特 性如何, 等等, 许多都是未知数。尤其是改革开放以 来, 进出口贸易以及国际间旅行等因素, 中国的外 来植物的增长可谓突飞猛进, 而其相关的研究则面 临巨大的挑战，任务非常繁重而且极其艰巨。

\section{3 中国外来入侵植物编目的主要进展}

近年来, 中国外来植物领域的编目有了长足发 展。《中国入侵植物名录》(马金双, 2013) 和《中国 外来入侵植物名录》(马金双和李惠茹, 2018) 相继出 版, 虽然以入侵为题, 但还包括了以往被认为是入 侵植物, 而实际上未达到入侵程度的归化植物和栽 培物种, 甚至还有一些是本土(国产)植物。对于以上 几类植物, 著作中均给予了澄清并分门别类进行记 载(极个别物种由于历史原因难以考证其来源, 认 知上可能略有差异), 并有中国省区市的分布记载。 在此基础上, 后续又有全国性分区域报道一 《中 国外来入侵植物调查报告》(马金双, 2014b)。再有, 最近刚刚出版的英文版《中国归化植物名录》(Yan et al., 2019), 既总结了前人的工作, 又将没有达到归 化程度的物种, 包括栽培种类以及国产种类等都予 以逐一澄清。此外, 中国栽培植物的工作也取得了 可喜的进展, 首部《中国栽培植物名录》顺利出版 (林秦文, 2018), 不仅对学名与物种进行了分类学考 证, 更列出了详细的来源地(洲际、国家或地区) 以及
在中国各省市区的具体分布等信息。最近出版的五 卷本《中国外来入侵植物志》(马金双, 2020), 基于 分类学的详细研究, 包括文献分析、野外采集以及 标本考证、入侵性质以及防范措施等, 为我们的工 作提供了较好的基础。

以上相关研究结果表明: 中国目前外来植物大 约有 1.4 万种 (不包括品种; 其中绝大部分为栽培植 物, 特别是室内栽培; 林秦文, 2018), 其中约有950 种为归化物种(Yan et al., 2019), 大约有400多种已 经具有入侵性质并造成危害(马金双，2020)。当然, 随着时间的推移以及研究的深入, 相关的数据会发 生变化, 包括每年新引入或进入的外来植物、新发 现的归化植物和入侵植物, 以及相关的分类学研究 结果的信息更新等。遗憾的是, 也许因为近年来对 植物分类学的关注度较低, 也许因为出版时间较短 或宣传不够, 这些资料并没有被学术界的一些相关 研究很好地参考使用; 有的工作虽然引证了以上部 分文献，但是并没有具体参考文献中的相关内容; 有的工作对存在不同意见或者处理的物种, 没有给 出作者选择的依据和理由。如: 许光耀等(2019)的 《中国归化植物组成特征及其时空分布》, 共记载 中国归化植物 1099 种, 其研究方法中明确指出, 数 据来源于《Flora of China》和《中国植物志》、相关 的标本馆和图像数据库, 以及各省植物志和全球外 来归化植物数据库。该文中的外来植物和归化植物 的定义与本文使用的定义一致, 但是, 其附录的 1099 种植物名录与上述的我们的研究结果出入较 大。李龙沁等(2021)的《中国西部地区归化植物时 空分布特征研究》, 使用的方法和数据与许光耀等 (2019)的相同, 记载的中国西部的归化植物仍然以 许光耀等(2019)的1 099种为基准, 尽管引证了 2019 年出版的相关工作(Yan et al., 2019), 但具体类群和 数据并没有变化。

\section{4 对近期两篇文章内容的商榷}

我们对许光耀等(2019)和李龙沁等(2021)文中 的外来归化物种数量进行了核对, 认为以下几方面 的问题需要订正(附详细的物种名单, 均以许光耀 等(2019)附录的1099种的学名字母为序, 不同的学 名, 等号后面的为接受名)。

\section{1 引种栽培植物被视为归化植物(共计123种)}

\subsection{1 经济作物(45种)}

以下物种是《中国植物志》(中英文版, 下同) 
等志书记载的栽培作物, 包括蔬菜、水果以及各类 经济作物, 虽然个别种类有时候会有逸生个体存在, 但如果没有人为的干预, 这些物种无法成功繁衍, 也不能在野外形成稳定的归化种群: 洋苟 (Allium cepa)、蒜(A. sativum)、圆滑番荔枝(Annona glabra)、 旱芹(芹菜)(Apium graveolens)、甜菜(Beta vulgaris)、 花椰菜 $($ Brassica cretica $=$ B. oleracea var. botrytis) 、 甘蓝(B. oleracea)、蔓菁(B. rapa)、依兰(Cananga odorata)、刀豆(Canavalia gladiata)、西瓜(Citrullus lanatus)、金鸡纳树 (Cinchona calisaya)、正鸡纳树 ( $C$. officinalis)、鸡纳树(C. pubescens)、大粒咖啡(Coffea liberica)、黄瓜(Cucumis sativus)、南瓜(Cucurbita moschata)、瓜儿豆(Cyamopsis tetragonoloba)、胡萝 卜(Daucus carota var. sativus)、甘薯(Dioscorea esculenta)、穇(Eleusine coracana)、树棉(Gossypium arboreum)、海岛棉(G. barbadense)、陆地棉(G. hirsutum)、烟草(Nicotiana tabacum)、橡胶树 (Hevea brasiliensis)、月桂(Laurus nobilis)、木犀榄(Olea europaea)、欧芹 (Petroselinum crispum)、棉豆 (Phaseolus lunatus)、胡椒(Piper nigrum)、踠豆(Pisum sativum)、黑刺李(Prunus spinosa)、草原樱桃(Prunus fruticosa)、四棱豆(Psophocarpus tetragonolobus)、 萝卜(Raphanus raphanistrum subsp. sativus $=R$. sativus (林秦文, 2018))、芸香(Ruta graveolens)、蓝胡 卢巴(Trigonella caerulea)、小黑麦(Secale sylvestre)、 阳芋(马铃薯) (Solanum tuberosum)、赤小豆(Vigna umbellata)、豇豆(V. unguiculata)、葡萄(Vitis vinifera)、 玉蜀㯟(Zea mays)、墨西哥玉米(Z. mexicana)等。

\subsection{2 观赏花卉(42种)}

以下观赏植物不但 《中国植物志》记载为栽培 种, 相关业界也都十分认可, 如果没有人为干预, 则无法繁衍, 不可能成为稳定的归化植物: 莳夢 (Anethum graveolens)、斑叶竹节秋海棠(Begonia maculata)、牛眼菊(Buphthalmum salicifolium)、兰花 美人蕉(Canna orchioides)、金盛菊(Calendula officinalis)、厚蓦凌霄(Campsis radicans)、黄花夹竹桃 (Cascabela thevetia = Thevetia peruviana) $、$ 吊兰 (Chlorophytum comosum)、多花贝母兰(Coelogyne venusta)、线姃菊 (Emilia coccinea)、花菱草 (Eschscholzia californica)、红雀珊瑚 (Euphorbia tithymaloides = Pedilanthus tithymaloides)，须苍石竹 (Dianthus barbatus)、香石竹(D. caryophyllus)、彩叶
万年青 (Dieffenbachia seguine)、缝线麻(Furcraea foetida)、非洲菊(Gerbera jamesonii)、细叶美女樱 (Glandularia tenera $=$ Cabrera tener $a$ 或者Verbena tenera)、辐射刺芙蓉(Hibiscus radiatus)、玫瑰茄( $H$. sabdariffa)、水鬼蕉(Hymenocallis littoralis)、茉莉花 (Jasminum sambac)、长寿花(Kalanchoe blossfeldiana)、大花紫薇(Lagerstroemia speciosa)、日本女贞 (Ligustrum japonicum)、贯月忍冬(Lonicera sempervirens)、人心果(Manikara zapota)、竹芋(Maranta arundinacea)、红纸扇(Mussaenda erythrophylla)、鬼 罂粟(Papaver orientale)、罂粟 $($ P. somniferum)、五星 花(Pentas lanceolata)、五彩苏(Plectranthus scutellarioides)、大花夏枯草(Prunella grandiflora)、蓝花 丹(Plumbago auriculata)、美丽决明(Senna spectabilis)、高雪轮(Silene armeria)、大蔓樱草(S. pendula)、 洬尾叶庭菖蒲(Sisyrinchium micranthum)、甜叶菊 (Stevia rebaudiana)、除虫菊 (Tanacetum cinerariifolium)、桂叶山牵牛(Thunbergia laurifolia)。

\subsection{3 其他栽培植物(36种)}

除上述的外来栽培植物外, 还有些种类实为栽 培植物, 许光耀等(2019) 作为归化植物进行统计, 但没有给出依据的文献: 软枝黄蝉(Allamanda $c a-$ thartica)、大叶南洋杉(Araucaria bidwillii)、簕竹 (Bambusa blumeana)、孝顺竹(B. multiplex)、苏木 (Caesalpinia sappan)、黄金树(Catalpa speciosa)、吉 贝(Ceiba pentandra)、线毛卷耳(Cerastium tomentosum)、日本扁柏(Chamaecyparis obtusa)、变叶木 (Codiaeum variegatum)、柠檬桉(Corymbia citriodora = Eucalyptus citriodora)、日本柳杉(Cryptomeria japonica)、纸莎草(Cyperus papyrus)、油棕(Elaeis guineensis)、美国白梣(Fraxinus americana)、红胶木 (Lophostemon confertus)、荷花木兰(Magnolia grandiflora)、五脉白千层(Melaleuca quinquenervia)、白 兰 $($ Michelia alba $=$ Michelia $\times$ alba $)$ 、芭蕉 $($ Musa bas$j o o$ )、欧洲夹竹桃(Nerium oleander)、稜稃雀稗 (Paspalum malacophyllum)、皱稃雀稗(P. plicatulum)、 加杨(Populus $\times$ canadensis)、牧豆树(Prosopis juliflora)、止痢蚤草(Pulicaria dysenterica)、毛洋槐 (Robinia hispida)、云木香(Saussurea costus = Aucklandia costus)、旋花羊角拗(Strophanthus gratus)、欧 丁香(Syringa vulgaris)、落羽杉 (Taxodium distichum)、池杉(T. distichum var. imbricatum)、墨西哥 
落羽杉(T. huegelii)、中间车轴草(Trifolium medium)、 磨擦草(Tripsacum laxum)、欧洲白榆(Ulmus laevis)。

\section{2 国产种类被作为归化植物(67种)}

许光耀等(2019)列出的归化植物中的一些种类 在中国的各类植物志中记载为国产, 作者没有提供 作为归化植物的文献, 如: 台湾相思(Acacia confusa)、异叶蒴莲 (Adenia heterophylla)、红豆冦 (Alpinia galanga)、糖胶树(Alstonia scholaris)、莲子 草(Alternanthera sessilis)、药葵(Althaea officinalis)、 皱缩链荚豆 (Alysicarpus rugosus)、耳基水苋 (Ammannia auriculata)、疮柄磨芋(Amorphophallus paeoniifolius)、日本黄花茅(Anthoxanthum odoratum subsp. nipponicum $=$ A. odoratum subsp. alpinum)、儿 茶(Acacia catechu)、金钮扣(Acmella paniculata)、西 伯利亚剪股颖 (Agrostis stolonifera)、大叶合欢 (Archidendron turgidum)、东方紫金牛(Ardisia elliptica)、芦竹(Arundo donax)、石习柏(Asparagus officinalis)、异苞滨藜(Atriplex micrantha)、木棉(Bombax ceiba)、头状沙拐疋(Calligonum caput-medusae)、青 葙 (Celosia argentea)、白花菜 $($ Cleome gynandra = Gynandropsis gynandra)、黄花草(Cleome viscosa $=$ Arivela viscosa)、红瓜(Coccinia grandis)、翼茎野百 合 (Crotalaria bialata $=$ 翅托叶猪屎豆C. alata)、莪术 (Curcuma zedoaria $=C$. phaeocaulis)、笔筒树 (Cyathea lepifera $=$ Sphaeropteris lepifera)、香附子 (Cyperus rotundus)、鸭茅(Dactylis glomerata)、缅甸 龙竹 (Dendrocalamus birmanicus)、美穗龙竹 (D. calostachyus)、黄竹(D. membranaceus)、高山发草 (Deschampsia atropurpurea) 、毛梗双花草 (Dichanthium aristatum)、海南大戟 (Euphorbia hainanensis)、泽漆(E. helioscopia)、对叶大戟 (E. sororia)、养麦(Fagopyrum esculentum)、圆雉石头花 (Gypsophila paniculata)、姜花(Hedychium coronarium)、毛果天芥菜(Heliotropium lasiocarpum)、睫苞 凤仙花(Impatiens bracteata)、土木香(Inula helenium)、欧洲菘蓝(Isatis tinctoria)、匙叶伽蓝菜 (Kalanchoe integra)、东亚女贞(Ligustrum obtusifolium subsp. microphyllum)、硬直黑麦草(Lolium rigidum)、兰屿百脉根 (Lotus taitungensis)、草龙 (Ludwigia hyssopifolia)、小苜宿(Medicago minima)、 锡金假鳞毛偋(Oreopteris elwesii)、酢浆草(Oxalis corniculata)、过江藤 (Phyla nodiflora)、海桐
(Pittosporum tobira)、草地早熟禾(Poa pratensis)、普 通早熟禾(P. trivialis)、小酸模(Rumex acetosella)、对 刺藤(Scutia myrtina)、棕叶狗尾草(Setaria palmifolia)、白玉草(Silene vulgaris)、新疆白芥(Sinapis arvensis)、亘荬菜(Sonchus wightianus)、金钮扣 (Spilanthes acmella =百能藏Blainvillea acmella)、大 泰竹(Thyrsostachys oliveri)、草莓车轴草(Trifolium fragiferum)、金慈姑(Typhonium roxburghii)、苦草 (Vallisneria natans)。

\section{3 引用植物学名存在误用、拼写错误及分类学的 名实问题(26种)}

许光耀等(2019) 文中注明植物的学名以 The Plant List (TPL, http://www.theplantlist.org/)确定，而 The Plant List是有文必录, 不能反映最新的研究进 展以及植物名称的科学性和准确性, 特别是中国的 外来植物领域。下列物种的学名已经被《Flora of China》(以下未标注文献者)和近年的相关研究作为 异名处理 (等号之后的为正名): 日本黄花茅 (Anthoxanthum odoratum subsp. nipponicum $=$ A. odo-

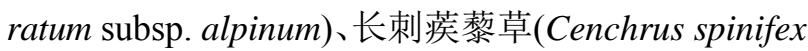
$=$ C. longispinus (马金双, 2020))、细叶黄皮(Clausena sanki $=$ C. anisum-olens (马金双, 2020))、白花菜 (Cleome gynandra $=$ Gynandropsis gynandra $)$ 、黄花 草 $($ Cleome viscosa $=$ Arivela viscosa)、柠檬桉 $($ Corymbia citriodora $=$ Eucalyptus citriodora $)$ 、翼茎 野百合(Crotalaria bialata=翅托叶猪屎豆C. alata)、 笔筒树 (Cyathea lepifer $=$ Sphaeropteris lepifera)、莪 术(Curcuma zedoaria $=$ C. phaeocaulis)、鸡冠刺桐 (Cylindrokelupha turgida) (中文和学名不匹配, 中文 对应的学名为 Erythrina crista-galli, 后者 Archidendron turgidum的中文应该是大叶合欢(林秦 文, 2018))、美洲沟繁缕(Elatine americana, 实为三 荵沟繁缕 E. triandra 的错误鉴定)、红雀珊瑚 (Euphorbia tithymaloides $=$ Pedilanthus tithymaloides)、橙红茴萝(Ipomoea cholulensis, 实为I. coccinea的错误鉴定(马金双, 2020))、蒿子杆(Ismelia carinata $=$ Glebionis carinata)、细果草龙 $($ Ludwigia ludwigia $=$ L. leptocarpa $($ 马金双, 2020) $)$ 、穗花赛葵 (Melochia spicata, 即许光耀等(2019)的Malvastrum americanum, 属于重复记录)、黄花月见草 $($ Oenothera erythrosepala $=$ O. glazioviana $) 、$ 多穗狼尾 草(Pennisetum polystachyo, 即牧地狼尾草学名的错 
误拼写, Pennisetum polystachion, 而且两者同时收 录)、葵叶茴萝 $($ Quamoclit sloteri = Ipomoea sloteri (Wood et al., 2020))、云木香(Saussurea costus = Aucklandia costus (Yan et al., 2019))、刺茄(Solanum surattense, 为牛茄子 $S$. capsicoides的错误鉴定, 刺 茄我国目前不产(马金双, 2020))、金钮扣(Spilanthes acmella =百能藏 Blainvillea acmella)、南洋楹 (Trigonella coerulea, 中文名和学名错误匹配: 按照 中文名应该是Albizia falcataria, 按照学名应该是蓝 胡卢巴(Trigonella caerulea)的错误拼写, 而且两者 同时记载)、郁金香(Tulipa gesneriana, 实为 $T$. iliensis 的错误鉴定)。另外, 冠䓫蔓锦葵(Andoda cristata)为 错误拼写, 应为Anoda cristata (Yan et al., 2019); 中 文名金钮扣出现两次, 但使用不同的学名, 即附录 第23种Acmella paniculata和第852种 Spilanthes acmella; 第 852 种的中文名应该是百能葳(Blainvillea acmella), Spilanthes acmella已经处理为异名。

\section{4 归化植物的引入时间考证不充分(若干种)}

许光耀等(2019)文中对部分归化物种引入时间 考证不充分。如日本黄花茅(Anthoxanthum odoratum subsp. nipponicum $=$ A. odoratum subsp. alpinum或者 A. nipponicum), 引入时间记载为 $2000 \mathrm{~s}$, 而该种在 东北早期的文献(1959和1995)就已经记载, 且为国 产种类(Yan et al., 2019); 莪术(Curcuma zedoaria), 引入时间记载为 $2000 \mathrm{~s}$, 所用的学名来自《中国植物 志》(中国植物志编辑委员会, 1981), 但该名称在英 文版《Flora of China》(Wu et al., 2000)中被认为是 一个错误鉴定的名称, 正确名称应该为 Curcuma phaeocaulis, 而本种在我国产云南(属于国产种), 栽 培于福建、广东、广西、四川, 在印度尼西亚及越 南也有分布; 再如假苍耳(Iva xanthiifolia)的引入时 间记载为 $1890 \mathrm{~s}$; 考证所有的标本与文献, 本种最早 在中国有记录并报道的时间为 1981 年(关广清, 1983); 还有细果草龙(Ludwigia ludwigia, 本种的学 名错写为属名的重复, 实际上本种学名应为 Ludwigia leptocarpa), 引入时间2000s, 但是本种在 国内最早的记录为2011年李宏庆等(SDP20689)所采 集的标本(现存于上海辰山植物标本馆, CSH), 此后 苗国丽等(2012)对该种进行了正式的报道。因篇幅 所限, 类似的例子不一一列出。

\section{5 已经归化的植物没有收录(若干种)}

据我们现有的资料对比分析，一些中国归化植
物许光耀等 (2019) 没有提及, 如直立孀泪花 (Tinantia erecta)、巴西商陆(Phytolacca thyrsiflora)、 少花龙葵(Solanum americanum)、田玄参 (Bacopa repens)、白籽草(Leucospora multifida)、矮小泥花草 (Micranthemum umbrosum)、腺萝鼠尾草(Salvia occidentalis)、糙毛狮牙苣(Leontodon hispidus)、合冠 鼠麦甸草 (Gamochaeta purpurea)、胶苑 (Grindelia squarrosa)、南美鬼针草(Bidens subalternans)、伏生 香檬菊(Pectis prostrata)、北美苍耳(Xanthium chinense) 等(未逐一列出, 详细内容请参见林秦文 (2018)和Yan等(2019))。

许光耀等(2019)的研究方法中说明该文的“归 化植物种类及分布数据分别来源于 《Flora of China》 (FOC, http://foc.iplant.cn/), 在线植物志(FRPS, http: //frps.iplant.cn), 自然标本馆(CFH, http://www.cfh.ac.cn/), 中国数字植物标本馆 $(\mathrm{CVH}, \mathrm{https}: / /$ www.cvh.ac.cn/), 中国植物图像库(PPBC, http://ppbc.iplant.cn/), 中华 本草(http://www.zhongyaocai360.com/zhonghuabencao/), 各省植物志, 全球外来归化植物数据库 (gloNAF, https://glonaf.org/)等数据库及其他文献资料”。其中 gloNAF是业内不错的信息源, 但是该数据基本上 是基于欧美等国家的信息, 正如许光耀等(2019)所 言, 有关中国的数据不仅有限而且很不准确, 其引 证中国数据的代表性论文(van Kleunen et al., 2015, 2019), 可靠性不高, 而且所依据的基本信息(Jiang et al., 2011)陈旧并缺乏权威性, 更不能代表中国目 前外来归化植物领域研究的最新成果。而中国的各 类植物志, 因为出版时间已经久远, 且受当时研编 条件的限制, 也已经不能反映中国植物的现状, 特 别是最近十几年外来植物的信息。因此, 相关人员 在使用相关资料和数据库时, 应尽量寻找最新的文 献和数据库, 采取分析的态度并审慎引用。

\section{5 中国外来归化植物研究展望}

众所周知，归化是入侵的前提。中国相关领域 研究任务艰巨: 首先, 整理相关志书并及时更新推 广, 保证研究中所使用数据的准确性与有效性, 才 能切实地推动中国外来植物的深入研究; 其次, 重 视野外实地调查, 从本底上研究清楚中国外来归化 植物的物种组成及入侵现状; 再次, 在大尺度上与 小尺度上同时开展中国外来入侵植物的入侵机制研 究，破解入侵机制的“黑匣子”; 第四，协调部门之 
间外来植物的管理机制，建立中国外来植物有效合 作平台，包括植物学及其相关的农林牧渔医药以及 有关的海关和检疫等部门; 第五, 积极开展国际合 作, 全面了解全球外来植物现状, 并重点布防中国 重要的潜在外来入侵植物。总之, 希望本文对中国 外来归化植物领域的研究有所借鉴或者帮助。

\section{参考文献}

Editorial Committee of Flora Reipublicae Popularis Sinicae (1959-2004). Flora Reipublicae Popularis Sinicae: Vol. 1-80. Beijing, Science Press. [中国植物志编辑委员会 (1959-2004). 中国植物志: 1-80卷. 北京, 科学出版社.]

Guan GQ (1983). A new invasive weed-Iva xanthifolia Nutt. Plant Quarantine, (5), 44-49. [关广清 (1983). 一种新侵 入我国的杂草一一假苍耳. 植物检疫, (5), 44-49.]

Jiang H, Fan Q, Li JT, Shi S, Li SP, Liao WB, Shu WS (2011). Naturalization of alien plants in China. Biodiversity and Conservation, 20, 1545-1556.

Li LQ, Xu GY, Gao Y, Mo XQ, Li HY (2021). Temporal and spatial distribution of naturalized plants in western China. Guihaia, [2021-03-26]. https://kns.cnki.net/kcms/detail/45. 1134.Q.20210324.1602.022.html. DOI: 10.11931/guihaia. gxzw202010052. [李龙沁, 许光耀, 高越, 莫训强, 李洪远 (2021). 中国西部地区归化植物时空分布特征研究. 广西 植物, [2021-03-26]. https://kns.cnki.net/kcms/detail/45.1134. Q.20210324.1602.022.html. DOI: 10.11931/guihaia. gxzw202010052.]

Lin QW (2018). Catalogue of Cultivate Plants in China. Science Press, Beijing. [林秦文 (2018). 中国栽培植物名 录. 科学出版社, 北京.]

Ma JS (2013). The Checklist of the Chinese Invasive Plants. Higher Education Press, Beijing. [马金双 (2013). 中国入 侵植物名录. 高等教育出版社, 北京.]

Ma JS (2014a). Current status and challenges of Chinese Plant Taxonomy. Chinese Science Bulletin, 59, 510-521. [马金双 (2014a). 中国植物分类学的现状与挑战. 科学通报, 59, 510-521.]

Ma JS (2014b). The Survey Reports on Chinese Alien Invasive Plants. Higher Education Press, Beijing. [马金双 (2014b). 中国外来入侵植物调研报告. 高等教育出版社, 北京.]

Ma JS (2020). Aline Invasive Flora of China: Vol. 1-5. Jiaotong University Press, Shanghai. [马金双 (2020). 中国外来入 侵植物志: $1-5$ 卷. 上海交通大学出版社, 上海.]

Ma JS, Li HR (2018). The Checklist of the Alien Invasive Plants of China. Higher Education Press, Beijing. [马金双, 李惠茹 (2018). 中国外来入侵植物名录. 高等教育出版社, 北京.]

Miao GL, Chen ZH, Xie WY, Ma K, Ma DD (2012). Four newly records of naturalized plant found in Zhejiang, China.
Journal of Zhejiang A\&F University, 29, 470-472. [苗国丽, 陈征海, 谢文远，马凯，马丹丹 (2012). 发现于浙江的4 种归化植物新记录. 浙江农林大学学报, 29, 470-472.]

Pyŝek P, Richardson DM, Rejmanek M, Webster GL, Williamson M, Kirschner J (2004). Alien plants in checklists and floras: towards better communication between taxonomists and ecologists. Taxon, 53, 131-143.

Pyŝek P, Pergl J, Eessl F, Lenzner B, Dawson W, Kreft H, Weigelt P, Winter M, Kartesz J, Nishino M, Antonova LA, Barcelona JF, Cabezas FJ, Cárdenas D, Cárdenas-Toro J, et al. (2017). Naturalized alien flora of the world: species diversity, taxonomic and phylogenetic patterns, geographic distribution and global hotspots of plant invasion. Preslia, 89, 203-274.

van Kleunen M, Dawson W, Essl F, Pergl J, Winter M, Weber E, Kreft H, Weigelt P, Kartesz J, Nishino M, Antonova LA, Barcelona JF, Cabezas FJ, Cárdenas D, Cárdenas-Toro J, et al. (2015). Global exchange and accumulation of non-native plants. Nature, 525, 100-103.

van Kleunen M, Pyŝek P, Dawson W, Essl F, Kreft H, Pergl J, Weigelt P, Stein A, Dullinger S, König C, Lenzner B, Maurel N, Moser D, Seebens H, Kartesz L, et al. (2019). The Global Naturalized Alien Flora (GloNAF) database. Ecology, 100, e02542. DOI: 10.1002/ecy.2542.

Wood JRI, Muñoz-Rodríguez P, Williams BRM, Scotland RW (2020). A foundation monograph of Ipomoea (Convolvulaceae) in the New World. PhytoKeys, 143, 1-823.

Wu ZY, Raven PH, Hong DY (1994-2013). Flora of China: Vol. 1-25. Science Press, Beijing, Science; Missouri Botanical Garden Press, St. Louis.

Wu SH, Hsieh CF, Rejmánek M (2004). Catalogue of the Naturalized Flora of Taiwan. Taiwania, 49, 16-31.

Xia CY, Fan XH, Li ZY, Wu XX, Pei ZX, Yu SX (2019). First interception of Croton bonplandianus Baill. from Rizhao, Shandong. Plant Quarantine, 33(4), 60-62. [夏常英, 范晓 虹, 李振宇, 吴学学, 裴紫馨, 于胜祥 (2019). 山东日照 新截获大戟科外来植物——本氏巴豆. 植物检疫, 33(4), 60-62.]

Xu GY, Li HY, Mo XQ, Meng WQ (2019). Composition and spatial-temporal distribution of Chinese naturalized plants. Chinese Journal of Plant Ecology, 43, 601-610. [许光耀, 李洪远, 莫训强, 孟伟庆 (2019). 中国归化植物组成特 征及其时空分布格局分析. 植物生态学报, 43, 601-610.]

Yan XL, Wang ZH, Ma JS (2019). The Checklist of the Naturalized Plants in China. Shanghai Science and Technology Press, Shanghai. 1-425. (in English) [间小玲, 王樟华, 马 金双 (2019). 中国归化植物名录. 上海科学技术出版社, 上海. 1-425.] 